\title{
Overcoming endocrine resistance in metastatic hormone receptor-positive breast cancer
}

\author{
Anishka D'Souza, Darcy Spicer and Janice Lu*
}

\begin{abstract}
Endocrine therapy has historically formed the basis of treatment of metastatic hormone receptor-positive breast cancer. The development of endocrine resistance has led to the development of newer endocrine drug combinations. Use of the CDK4/6 inhibitors has significantly improved progression-free survival in this group of patients. There are multiple studies of the use of P13K inhibitors and mTOR inhibitors for use as subsequent lines of therapy, particularly for endocrine resistance. The optimal sequencing of therapy should be based on medical comorbidities, prior adjuvant therapies, quality of life, side-effect profile, and disease-free interval.
\end{abstract}

\section{Background}

Breast cancer is the most frequently diagnosed cancer in women. Around $5-10 \%$ of cases are metastatic at diagnosis, and close to $30 \%$ of patients with early stage disease will go on to relapse with metastatic disease [1]. Hormone receptor-positive breast cancer makes up 70\% of breast cancers cases. Endocrine therapy remains the mainstay of early treatment. A significant number of these patients will develop either primary or secondary endocrine resistance, prompting the need for newer treatment options [2].

\section{Endocrine therapies}

Tamoxifen has been used in the management of metastatic hormone receptor-positive breast cancer for decades. The third-generation aromatase inhibitors (AIs) are used in both the first- and second-line settings in the management of hormone receptor-positive metastatic breast cancer.

Fulvestrant is an selective estrogen receptor downregulator (SERD) used in the management of metastatic hormone receptor-positive breast cancer in both the first- and subsequent-line settings. The $500 \mathrm{mg}$ fulvestrant dose was approved based on the results of the CONFIRM trial, which showed improvement in both

* Correspondence: Janice.Lu@med.usc.edu

USC Norris Comprehensive Cancer Center, 1441 Eastlake Avenue, LoS Angeles, CA 90033, USA

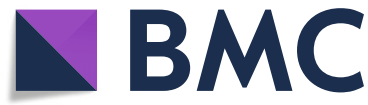

progression-free and overall survival with the 500-mg dose compared with the 250-mg dose [3]. The FIRST trial compared the use of fulvestrant $500 \mathrm{mg}$ monthly with anastrazole $1 \mathrm{mg}$ daily in postmenopausal women with advanced or metastatic hormone receptor-positive breast cancer. This study demonstrated a significant improvement in time to progression and an improved overall survival in the fulvestrant compared with the anastrazole group $[4,5]$. The FALCON trial further assessed the progression-free survival advantage observed in the FIRST study. This was a phase III study comparing the use of fulvestrant $500 \mathrm{mg}$ monthly with anastrazole $1 \mathrm{mg}$ daily in endocrine therapy-naïve, postmenopausal patients with metastatic hormone receptorpositive breast cancer [6]. A total of 462 patients were randomized to treatment. Median progression-free survival was 16.6 months with fulvestrant and 13.0 months with anastrazole $(P=0.0486)$. The objective response rate in patients with measurable disease was 46 and $45 \%$, respectively, in patients treated with fulvestrant and anastrazole. The median duration of response was longer in the group treated with fulvestrant (20.0 months) compared with that in the anastrazole group (13.2 months). Data is as yet immature for evaluation of overall survival. The rates of adverse events were similar in the fulvestrant (73\%) and anastrazole (75\%) groups, with the rates of serious adverse events in both groups being $13 \%$. Overall, the results of the FALCON study support the superior efficacy of fulvestrant

(c) The Author(s). 2018 Open Access This article is distributed under the terms of the Creative Commons Attribution 4.0 International License (http://creativecommons.org/licenses/by/4.0/), which permits unrestricted use, distribution, and reproduction in any medium, provided you give appropriate credit to the original author(s) and the source, provide a link to the Creative Commons license, and indicate if changes were made. The Creative Commons Public Domain Dedication waiver (http://creativecommons.org/publicdomain/zero/1.0/) applies to the data made available in this article, unless otherwise stated. 
over anastrazole in the treatment of postmenopausal women with hormone receptor-positive metastatic breast cancer who did not receive previous endocrine therapy. In addition, patients with disease confined to the bone tended to do better with fulvestrant therapy. Fulvestrant is FDA-approved for use in both endocrine-resistant and naïve settings.

\section{Resistance to endocrine therapy}

Endocrine resistance is a major clinical problem encountered in the treatment of breast cancer. Endocrine resistance can be divided into two groups. Primary endocrine resistance is defined as relapse during the first 2 years of adjuvant endocrine therapy or progressive disease within the first 6 months of first-line endocrine therapy for metastatic breast cancer. Secondary resistance is defined as relapse while on adjuvant endocrine therapy but after the first 2 years of treatment, relapse within 12 months of completing adjuvant endocrine therapy, or progressive disease six or more months after starting endocrine therapy for metastatic breast cancer [7].

There are a number of different mechanisms implicated in the development of endocrine resistance. Loss of estrogen receptor (ER) expression is one possible cause, with between 10 and 20\% of initially ER-positive patients converting to negative on relapse [8]. Another mechanism being explored is the development of endocrine receptor mutations [9]. Variations in tamoxifen metabolism have been studied as a possible cause of differing responses to tamoxifen therapy. The CYP2D6 gene product is responsible for the metabolism of tamoxifen to endoxifen, one of its active metabolites. The drug metabolism varies based on the CYP2D6 allelic profile, with certain genotypes associated with a higher rate of relapse [8]. Growth factors, such as EGFR, insulin/IGFs, and FGFR, and their respective signaling pathways have also been studied as potential mechanisms of endocrine resistance [9]. Studies suggest that HER2/neu overexpression is associated with poorer response to tamoxifen therapy. HER2 may lead to tamoxifen resistance by activating estrogen receptor co-activator proteins [8]. P13K/AKT/mTOR signaling pathway activation plays a major role in the development of endocrine resistance and is a target of many therapies designed to overcome resistance [10]. There are also studies evaluating the role of the ESR1 mutation in acquired endocrine resistant breast cancer. There is an ongoing phase II study evaluating the efficacy of fulvestrant in patients with ESR1-mutated breast cancer (NCT03202862).

\section{CDK4/6 inhibitors}

\section{Mechanism of action}

Cyclin-dependent kinases (CDKs) 4 and 6 promote entry into the cell cycle through the phosphorylation of the retinoblastoma protein $(\mathrm{Rb})$ [11]. This leads to the transition from gap 1 (G1) to the DNA synthesis (S) phase of the cell cycle, ultimately leading to cell division [12]. Activation of the pathway leading to Rb phosphorylation has been associated with the development of endocrine resistance [10]. CDK 4/6 inhibitors block the phosphorylation of $\mathrm{Rb}$, leading to cell cycle arrest, and can reverse endocrine resistance when used. Three CDK $4 / 6$ inhibitors currently being used in the treatment of advanced breast cancer are palbociclib, ribociclib, and abemaciclib (Table 1) [13, 14].

\section{Palbociclib}

Palbociclib is an oral, selective inhibitor of CDK $4 / 6$ approved for use in the first- and second-line settings for advanced or metastatic hormone-receptor positive breast cancer.

PALOMA-2 was a phase III study of palbociclib and letrozole as first-line therapy for postmenopausal women with estrogen-receptor (ER)-positive, HER2-negative advanced breast cancer [15]. A total of 666 women were randomly assigned, in a 2:1 ratio, to receive either palbociclib $125 \mathrm{mg}$ administered in 4-week cycles (3 weeks on, 1 week off) or placebo, in combination with continuous daily letrozole $2.5 \mathrm{mg}$. The median age of patients was 62 years in the palbociclib-letrozole group and 61 years in the placebo-letrozole group. Of all patients, $37.2 \%$ had newly diagnosed metastatic breast cancer, $40.7 \%$ had a disease-free interval of more than 12 months, and $22.1 \%$ had a disease-free interval of less than 12 months. The median progression-free survival was 24.8 months in the palbociclib group and 14.5 months in the control group (HR 0.58; 95\% CI, $0.46-0.72$; two-sided $P<0.001$ ). Data from longer-term follow-up of the PALOMA-2 trial were presented at the 2017 San Antonio Breast Cancer Symposium and revealed a median PFS of 27.6 months in the palbociclib/ letrozole arm at 38 months follow-up and 14.5 months in the placebo arm at 37 months follow-up [16]. The objective response rates among all patients randomly assigned to receive palbociclib-letrozole were $42.1 \%$ (95\% CI, 37.5-46.9) compared with 34.7\% (95\% CI, 28.4-41.3) in patients randomized to receive placebo. At the time of publication, the overall survival data was still immature. The most common adverse events in the study group were neutropenia, leucopenia, fatigue, nausea, arthralgia, and alopecia. Grade 3 or 4 hematologic events included neutropenia $(66.4 \%$ of patients in palbociclib-letrozole group vs. $1.4 \%$ of patients in the placebo-letrozole group), leucopenia ( 24.8 vs. $0 \%)$, anemia (5.4 vs. $1.8 \%)$, and thrombocytopenia (1.6 vs. $0 \%)$. Nonhematologic adverse events included fatigue, nausea, and arthralgia.

PALOMA-3 was a phase III study of the use of palbociclib in previously treated patients with advanced 
Table 1 Summary of trials using CDK4/6 inhibitors in patients with metastatic hormone receptor-positive breast cancer

\begin{tabular}{lllll}
\hline Agent & ET/Setting & Median PFS & HR & Trial \\
\hline Palbociclib & Letrozole, 1st-line & 24.8 vs 14.5 months & 0.58 & PALOMA-2 \\
Palbociclib & Fulvestrant, 2nd-line & 9.2 vs 3.8 months & 0.42 & PALOMA-3 \\
Ribociclib & Letrozole, 1st-line & 25.3 vs 16.0 months & 0.56 & MONALEESA-2 \\
Abemaciclib & Fulvestrant, 2nd-line & 16.4 vs 9.3 months & 0.55 & MONARCH-2 \\
\hline
\end{tabular}

$E T$ endocrine therapy, PFS progression-free survival

hormone-receptor positive, HER2-negative breast cancer [11]. Patients were eligible if their cancer had relapsed or progressed with prior endocrine therapy. Both preand postmenopausal women were allowed to participate. A total of 521 patients were randomly assigned in a 2:1 ratio to receive palbociclib (125 mg daily 3 weeks on and 1 week off cycles) or matching placebo in addition to fulvestrant (500 mg every 14 days for 3 doses followed by every 28 days thereafter). The median age of all patients was 57 years, with the majority of patients (79.3\%) being postmenopausal. Of all patients enrolled, 23.4\% had metastatic disease at initial diagnosis. The median progression-free survival was 9.2 months in the palbociclib-fulvestrant group and 3.8 months in the placebo-fulvestrant group (HR 0.42; 95\% CI, 0.32-0.56; $P<0.001)$. The relative differences in PFS were similar between the pre- and postmenopausal groups. The objective response rate was higher in the study versus the placebo group; however, this difference was not statistically significant $(10.4$ vs. $6.3 \%, P=0.16)$. Grade 3 or 4 hematologic toxicities were all more frequent in the palbociclib-fulvestrant group when compared with the placebo-fulvestrant group (neutropenia 62.0 vs. 0.6\%, leucopenia 25.5 vs. $0.6 \%$, anemia 2.6 vs. $1.7 \%$, and thrombocytopenia 2.3 vs. $0 \%$ ). The most frequent nonhematologic toxicities were fatigue, nausea, and headache.

The PEARL study is a phase III trial comparing the use of palbociclib combined with endocrine therapy (exemestane or fulvestrant) with chemotherapy (capecitabine) in patients with $\mathrm{HR}+$ /HER2- metastatic breast cancer who are resistant to a nonsteroidal aromatase inhibitor [17].

The POLARIS trial is a prospective, non-interventional study of 1500 patients who were treated with palbociclib in the USA and Canada. The study will evaluate the prescribing and treatment patterns of the patients; assess the clinical response to palbociclib; perform biomarkers studies to help elucidate potential mechanisms of response and resistance to palbociclib; assess patient quality of life, study survival, and toxicity of the drug; and evaluate sequencing of treatments in these patients [18].

\section{Ribociclib}

Ribociclib (LEE011; Novartis) is a selective inhibitor of CDK 4/6 currently being studied for use in the treatment of pre- and postmenopausal women with advanced hormone-receptor positive breast cancer.

Based on the results of the phase III MONALEESA-2 trial, the FDA approved its use in combination with letrozole as first-line endocrine therapy in postmenopausal patients with hormone receptor-positive breast cancer [19]. Patients were postmenopausal with locally recurrent or metastatic hormone receptor-positive, HER2-negative breast cancer who had not previously received systemic therapy for their advanced disease. A total of 668 patients were randomized to receive ribociclib (600 mg per day on a 3-week on and 1-week off schedule) plus letrozole ( $2.5 \mathrm{mg}$ per day continuously) or placebo plus letrozole. The median age of patients was 62 years, with $34.0 \%$ having advanced or metastatic disease at initial diagnosis. After a median follow-up of 26.4 months, the median progression-free survival was 25.3 months in the ribociclib group and was 16.0 months in the placebo group ( $\mathrm{HR} 0.56 ; 95 \% \mathrm{CI}, 0.43-0.72$; $\left.P=3.29 \times 10^{-6}\right)$. At 12 months, the progression-free survival was $72.8 \%$ in the ribociclib group and $60.9 \%$ in the placebo group. At 18 months, the progression-free survival was 63.0 and $42.2 \%$, respectively. The overall response rates were $40.7 \%$ in the ribociclib group and $27.5 \%$ in the placebo group in the intention-to-treat population. At the time of the interim analysis, overall survival results were not mature. Grade 3 or 4 adverse events included neutropenia (59.3\% in the ribociclib group vs. $0.9 \%$ in the placebo group), leucopenia (21.0 vs. 0.6\%), hypertension (9.9 vs. $10.9 \%$ ), and elevated alanine aminotransferase level (9.3 vs. 1.2\%). QTc interval prolongation of at least $60 \mathrm{msec}$ from baseline occurred in 9 patients in the ribociclib group $(2.7 \%)$ and no patients in the placebo group.

The MONALEESA-3 trial (NCT02422615) is currently ongoing. This study is evaluating the use of ribociclib in combination with fulvestrant in patients with advanced breast cancer who have received one prior line of endocrine therapy.

The phase III MONALEESA-7 trial (NCT02278120) is specifically designed for pre- or perimenopausal women with advanced hormone receptor-positive breast cancer. Patients were randomized to receive tamoxifen or a non-steroidal aromatase inhibitor (letrozole or anastrozole) with goserelin in combination with ribociclib or with placebo. Progression-free survival was significantly improved 
in the ribociclib arm (23.8 months) versus the placebo arm (13.0 months) [20].

\section{Abemaciclib}

Abemaciclib (LY2835219) is the third CDK 4/6 inhibitor currently being studied for use in hormone receptorpositive advanced breast cancer. It is unique from palbociclib and ribociclib in that it can be dosed continuously and has a higher response rate when used as monotherapy $[12,13]$. It also demonstrates greater selectivity for CDK4 than for CDK6. In the phase I study investigating the use of the drug in patients with various solid tumors, the dose-limiting toxicity was diarrhea, which is different from the DLT of bone marrow suppression seen with the other CDK4/6 inhibitors [21]. Abemaciclib was recently FDA-approved for the use in combination with fulvestrant in the management of hormone receptor-positive, HER2-negative metastatic breast cancer after progression on endocrine therapy. It is also approved as monotherapy in patients with hormone receptor-positive/HER2- metastatic breast cancer who had progressed during or after treatment with endocrine therapy and chemotherapy.

The use of abemaciclib monotherapy is based on the results of the MONARCH-1 trial [22]. This was a phase II, single-arm study of the use of abemaciclib monotherapy in women with hormone receptor-positive, HER2-negative metastatic breast cancer who have progressed on endocrine therapy or chemotherapy. A total of 132 patients were treated with abemaciclib monotherapy on the study, with a median of 3 prior lines of therapy for advanced disease. At the 8-month interim analysis, the overall response rate was $17.4 \%$ and the median progression free survival was 5.7 months.

Support for the use of the abemaciclib and fulvestrant combination came from the results of the phase III MONARCH-2 study [23]. This was a randomized trial comparing the use of fulvestrant with or without abemaciclib in patients with hormone receptor-positive, HER2-negative advanced breast cancer who had progressed on endocrine therapy. A total of 669 patients were randomized. The median progression-free-survival was 16.4 months in the combination group, compared with 9.3 months in the fulvestrant-only group (HR, 0.553 ; 95\% CI, $0.449-0.681 ; P<.001)$. The overall response rate was $35.2 \%$ in the abemaciclib group, with 14 patients $(3.1 \%)$ attaining a complete response. This compared with an ORR of $16.1 \%$ in the control arm and one CR (0.4\%). Overall survival data was not yet mature at the time of data cutoff. The most commonly occurring adverse events were neutropenia, diarrhea, fatigue, nausea, and abdominal pain, the majority of which were grade 1 or 2 . Serious adverse events occurred in $22.4 \%$ of patients in the combination arm and $10.8 \%$ of patients in the control group.
The MONARCH-3 study evaluates the use of abemaciclib with letrozole as first-line treatment in postmenopausal women with metastatic hormone receptor-positive breast cancer [24]. Results of the trial interim analysis were presented at ESMO this year and demonstrated a significant improvement in progression-free survival with a hazard ratio of $0.543(p=0.000021)$. The objective response rate in patients with measurable disease was $59 \%$ in the abemaciclib arm compared with $44 \%$ in the control arm.

\section{CDK4/6 resistance}

Proposed mechanisms of resistance to CDK4/6 inhibitors include the MAPK and PI3K pathways, mutations or deletions of RB1, and amplification or overexpression of cyclin E1.

\section{mTOR inhibitors}

The PI3K/AKT/mTOR pathway is dysregulated in many types of cancers. It is regulated at different points by multiple tumor suppressor and oncogenes, which provide sites of potential mutations in the pathway [25]. P13K alterations are common in many cancers, with activating mutations of the P1K3CA gene commonly found in breast cancer. Other common mutations in the pathway found in breast cancer include mutated AKT and loss of PTEN [26]. Dysregulation in the mTOR pathway have been implicated in the development of endocrine resistance in breast cancer, making this an attractive target for therapy [2]. Everolimus is a rapalogue or rapamycin analogue that acts as a potent inhibitor of mTOR. It has been the focus of a variety of trials for the use in hormone receptor-positive breast cancer.

The TAMRAD study was a phase II trial evaluating the use of everolimus in combination with tamoxifen in postmenopausal women with metastatic hormone receptor-positive, HER2-negative breast cancer who had progressed on prior aromatase inhibitor therapy [27]. The clinical benefit rate at 6 months was $61 \%$ in the everolimus arm and $42 \%$ in the tamoxifen only arm $(P=0.045)$. The time to progression was also significantly improved in the everolimus group (8.6 vs. 4.5 months; $P=0.002)$. There was a $46 \%$ reduction in the risk of progression and $55 \%$ reduction in the risk of death associated with the everolimus-tamoxifen combination. On subgroup analysis, it appeared that the benefit of the combination therapy was mainly for patients who had secondary endocrine resistance.

The BOLERO-2 study evaluated the use of an everolimus-exemestane combination in the treatment of hormone receptor-positive, HER2-negative metastatic breast cancer [28]. In 2012, the FDA approved the use of the combination in this group of patients who had progressed on prior aromatase inhibitors. In the phase III trial, 
postmenopausal women with ER-positive, HER2nonamplified advanced breast cancer who had progressed on prior aromatase inhibitors were randomized in a 2:1 ratio to receive oral everolimus ( $10 \mathrm{mg}$ daily) or placebo, in combination with exemestane ( $25 \mathrm{mg}$ daily). A total of 724 women were randomized, with a median age of 62 years. Fifty-six percent of the patients had visceral involvement of their cancer and $76 \%$ had bone metastases. The median progression-free-survival was 6.9 months in the everolimus arm and 2.8 months in the placebo arm (HR 0.43; 95\% CI, 0.35-0.54; $P<0.001$ ). Median overall survival was not significant between the two groups (31.0 months in the everolimus-exemestane group and 26.6 months in the placebo-exemestane group; $P=0.1426$ [29]. The most common grade 3 or 4 adverse events were stomatitis, dyspnea, hyperglycemia, fatigue, and pneumonitis, all of which were more common in the everolimus arm.

The BOLERO-4 study (NCT01698918) is a currently ongoing trial evaluating the use of everolimus in combination with letrozole as first-line therapy for postmenopausal women with hormone receptor-positive, HER2-negative metastatic breast cancer.

The BALLET trial was a single-arm European phase IIIb study evaluating the safety of everolimus plus exemestane in post-menopausal women with hormone receptor-positive, HER2-negative advanced or metastatic breast cancer who had progressed on prior non-steroidal aromatase inhibitors [30]. A total of 2133 patients were enrolled in the study and were treated with daily doses of everolimus (10 mg per day) and exemestane $(25 \mathrm{mg}$ per day). The median age of patients enrolled was 63 years. The majority of patients (65\%) received everolimus/exemestane as a third or higher line of therapy. Overall, $42.7 \%$ of patients experienced grade $3 / 4$ adverse events, with the majority of these being attributed to the everolimus. At the time of study analysis, 121 (5.7\%) on-treatment deaths were documented, with 66 (3.1\%) of these attributed to disease progression and $46(2.2 \%)$ attributed to adverse events.

The MANTA trial is a four-arm, phase II study of fulvestrant + continuous AZD2014 (vistusertib), fulvestrant + intermittent vistusertib, fulvestrant + everolimus, and fulvestrant alone in women with estrogen receptor-positive, HER2-negative advanced or metastatic breast cancer [31]. AZD2014 is a novel inhibitor of mTORC1 and mTORC2. The everolimus/fulvestrant arm demonstrated improved PFS compared with vistusertib/fulvestrant (12.3 vs 7.6 months) and with fulvestrant alone (12.3 vs 5.4 months). The objective response rate was also higher in the everolimus/fulvestrant group (41\%) when compared with the fulvestrant alone (25.0\%), fulvestrant/vistusertib continuous (30.4\%), and fulvestrant/vistusertib intermittent $(28.6 \%)$ groups.

\section{$\mathrm{HR}+$ and HER2+}

For HER2/HR-positive patients, treatment with HER2targeted therapy in combination with chemotherapy is the most common first-line therapy. There are no trials directly comparing the use of endocrine plus HER2-targeted therapy with the use of chemotherapy combined with HER2-targeted agents. Treatment with a HER2-targeted agent in combination with endocrine therapy can be considered in certain patients, in whom treatment with chemotherapy is not immediately indicated.

Studies of mouse models indicate that the cyclin D1-CDK4 pathway may play a role in the development of resistance to HER2-directed therapies in patients with HER2-positive breast cancer. The use of CDK4/6 inhibitors in this population may resensitize these patients to anti-HER2 therapy. The monarcHER study is a randomized, multicenter, phase 2 trial comparing the use of abemaciclib and trastuzumab with or without fulvestrant to physician's choice standard-of-care chemotherapy plus trastuzumab in postmenopausal women with locally advanced or metastatic HR+/HER2+ breast cancer. Participants must have received at least two lines of HER2-directed therapy prior to enrollment, including trastuzumab emtansine (T-DM1) in any disease setting. Patients must not have received prior treatment with CDK4/6 inhibitors. The study is currently ongoing. The PATRICIA trial is an ongoing multicenter phase II trial evaluating the use of the combination of palbociclib plus trastuzumab, with or without letrozole, in post-menopausal women with HER2-positive advanced or metastatic breast cancer previously treated with chemotherapy and trastuzumab. The study includes three treatment arms. Arm A includes patients with ER-/HER2+ disease who will receive palbociclib and traztuzumab. Arms B2 and B2 include patients with ER+/HER2+ disease who will receive palbiciclib plus trastuzumab and palbocilcib/ traztuzumab/letrozole, respectively. Another study evaluates the use of tucatinib, a novel HER2-targeted tyrosine kinase inhibitor, in combination with palbociclib and letrozole as a first- or second-line therapy option for patients with HER2+/ER+ metastatic breast cancer.

\section{P13K inhibitors \\ Mechanism of action}

As mentioned previously, the P13K-AKT-mTOR pathway is a frequently activated signaling pathway in breast cancer. The P13K pathway may be involved in resistance to cancer therapies, including endocrine therapy, chemotherapy, and targeted drugs. Inhibition of P13K may help restore sensitivity to other therapies when use in combination regimens. A number of P13K inhibitors are under investigation for treatment of several malignancies, including breast cancer (Table 2). 
Table 2 Summary of trials combining endocrine therapy with PI3K inhibitors in patients with metastatic hormone receptor-positive breast cancer

\begin{tabular}{llll}
\hline Agent & ET & Setting & Trial \\
\hline Alpelisib & Fulvestrant & After progression on Al & SOLAR-1; NCT02437318 \\
Buparlisib & Fulvestrant & After progression on Al & BELLE-2; NCT01610284 \\
Buparlisib & Fulvestant & After progression on mTOR inhibitor & BELLE-3; NCT01633060 \\
Pictilisib & Fulvestrant & After progression on Al & FERGl; NCT01437566 \\
Pictilisib + Palbociclib & Fulvestrant & After progression on Al & PASTOR; NCT02599714 \\
Gedatolisib + Palbociclib & Fulvestrant or Letrozole & 3 arms: & NCT02684032 \\
& & - No prior endocrine therapy & \\
& & - Progression on or after 1 line of endocrine therapy, & \\
& & no prior CDK inhibitor therapy & \\
& following prior CDK inhibitor therapy &
\end{tabular}

ET endocrine therapy, $A l$ aromatase inhibitor

\begin{abstract}
Alpelisib
Alpelisib has demonstrated promising early efficacy in studies, both as a single agent and in combination with fulvestrant [32-34]. Data presented by Juric et al. demonstrated an improved disease control rate and clinical benefit rate in patients with P13KCA-mutations, compared with no response in those with wild-type tumors. The SOLAR-1 trial is an ongoing phase III study of the use of alpelisib combined with fulvestrant in men and postmenopausal women with ER-positive/HER2-negative breast cancer which progressed on or after treatment with an aromatase inhibitor.
\end{abstract}

\section{Buparlisib}

Buparlisib is a pan-P13K inhibitor that inhibits all four of the class $1 \mathrm{P} 13 \mathrm{~K}$ isoforms [35]. The BELLE-2 trial was a phase III study evaluating the use of buparlisib plus fulvestrant in post-menopausal women with hormone receptor-positive, HER2-negative advanced or metastatic breast cancer which had progressed on an aromatase inhibitor [36]. A total of 1147 women were randomized to receive either a buparlisib/fulvestrant combination or fulvestrant monotherapy. There was a significant improvement in median PFS observed in the buparlisib arm compared with the fulvestrant arm (6.9 vs 5.0 months). Among patients with known P13K pathway status, median PFS in the combination and control arm was 6.8 and 4.0 months, respectively. There was no significant difference in PFS between the treatment arms in patients without P13K-mutations. Overall survival data was immature at the time of study assessment. Serious adverse events occurred in $23 \%$ of patients treated in the bupalisib arm compared with $16 \%$ in the control arm, the most common of which were elevations in AST and ALT and hyperglycemia.

The BELLE-3 trial evaluated the use of combination treatment with buparlisib and fulvestrant in patients with HR-positive/HER2-negative MBC who had progressed on or after treatment with an mTOR inhibitor [37]. Similar to the BELLE-2 study, there was an improvement in median PFS in the bupalisib arm compared with the control arm (3.9 vs 1.8 months). Toxicity data was similar to that of the BELLE-2 study.

\section{Pictilisib}

The FERGI trial was a two-part phase 2 study of the use of pictilisib plus fulvestrant in post-menopausal women with ER+/HER2- advanced or metastatic breast cancer resistant to treatment with an aromatase inhibitor [38]. Patients were randomized to receive either pictilisib plus fulvestrant or placebo plus fulvestrant. Part 1 of the study included patients with and without P13K mutations, while part 2 only included patients with P13K mutations. No difference in PFS was found between treatment arms in either part 1 or part 2 of the study. This lack of improvement in PFS may in part be due to significant toxicity associated with pictilisib use, leading to many patients not receiving the full dose of the drug until progression. Forty (45\%) of patients in part 1 had dose modifications. The pictilisib dose was reduced for part 2 of the study; however, $17 \%$ of patients still required dose reductions due to toxicity.

\section{Taselisib}

Taselisib is an investigational $\mathrm{P} 13 \mathrm{~K}$ being studied in combination with fulvestrant in patients with advanced or metastatic breast cancer who have progressed or recurred during or after treatment with an aromatase inhibitor (NCT02340221).

\section{Combined CDK4/6 and mTOR inhibition}

The interaction between the CDK4/6 and P13K/mTOR/ AKT pathways is thought to play an important role in endocrine receptor-positive breast cancer. Studies show that CDK4/6 resistant cell lines remain sensitive to mTORC $1 / 2$ inhibition, suggesting that combining these 
therapies may be an option for patient who has relapsed while on CDK4/6 therapy. Studies are being conducted studying novel combinations of P13K and CDK4/6 inhibitors with endocrine therapy [39].

The PASTOR trial is a phase I/II multicenter trial of the combination of vistusertib, palbociclib, and fulvestrant in postmenopausal patients with advanced or metastatic endocrine receptor-positive breast cancer. There is also an ongoing phase Ib study of the use of gedatolisib, a potent dual $\mathrm{P} 13 \mathrm{~K} / \mathrm{mTOR}$ inhibitor, in combination with palbociclib/fulvestrant and with palbociclib/letrozole [40].

\section{Summary}

There are multiple ongoing trials combining PI3K inhibitors with other agents, such as CDK4/6 inhibitors and endocrine therapy. Future therapy may evaluate the use of these drugs with inhibitors to other pathways, such as STAT3, MYC, MEK, and PARP.

\section{Third- and fourth-line therapies}

High-dose estrogens and progestins can be considered as late-line options for therapy in metastatic hormone-receptor positive breast cancer. Megesterol acetate and medroxyprogesterone are progestins with activity in metastatic breast cancer. A study of postmenopausal women with locally advanced or metastatic breast cancer who had progressed on first-line tamoxifen demonstrated an overall response rate of $25 \%$ with megesterol acetate and 43\% with medroxyprogesterone [41]. Median progression-free survival was 15 months for megesterol and 10 months for medroxyprogesterone. A dose-escalation trial of megesterol acetate demonstrated a higher toxicity rate without any improvement in effectiveness with the higher doses [42]. Therefore, the $160 \mathrm{mg}$ daily dose is generally used. Estrogen compounds have also been used in the management of metastatic breast cancer. A study of 32 patients with endocrine therapy-resistant disease treated with diethylstilbestrol (DES) yielded an objective response rate of $31 \%$ [43]. Patients had received an average of 4 prior endocrine therapies and 1 prior chemotherapy. A phase II study evaluating the use of 6 versus $30 \mathrm{mg}$ of estradiol in patients with advanced breast cancer showed that the lower dose provided a similar clinical outcomes as the $30 \mathrm{mg}$ dose. The lower dose was also associated with fewer adverse events.

\section{Optimal sequence of therapy}

There are many options in the sequencing of therapy for endocrine receptor-positive, metastatic breast cancer in post-menopausal women. Although first-line treatment with a CDK/4/6 inhibition has significant improvement in PFS, the total PFS is similar regardless of the sequencing (Fig. 1a, b). Treatment decisions should be based on medical comorbidities, prior adjuvant therapies, and disease-free interval [44]. First-line treatment with an aromatase inhibitor or fulvestrant are still viable options and offer a PFS of 14 and 16.6 months, respectively. Frontline use of the combination of a CDK4/6 inhibitor with an aromatase inhibitor, such as palbociclib/ letrozole and ribociclib/letrozole, offer a greater than 24-month PFS. Subsequent-line therapies include the use of palbociclib or abemaciclib with fulvestrant, the combination of everolimus with exemestane, and single-agent abemaciclib. The use of PI3K-inhibitors both single-agent and in combination with fulvestrant are being studied for use in patients with endocrine-resistant disease. Immunotherapy and CAR-T therapy are also being explored as other options of treatment. The use of biomarkers, including ESR1 mutation, and genomic profiling may provide useful future tools to direct therapy.






\section{Future directions}

There are several ongoing studies of the use of combinational treatments including PD-1 and PD-L1 inhibitors in breast cancer treatment $[45,46]$. The JPCE trial included 28 patients with hormone receptor-positive, HER2-negative metastatic breast cancer treated with abemaciclib and pembrolizumab. The PR rate at 16 weeks was $14.3 \%$, and the overall response rate at 16 -week analysis was $14.3 \%$. Another ongoing trial assesses the combination of pembrolizumab, letrozole, and palbociclib in postmenopausal patients with metastatic estrogen receptor-positive breast cancer who did not respond to letrozole and palbocilcib.

There are multiple ongoing studies of the use of CAR-T therapy for patients with breast cancer. Another means of treatment being evaluated for use in solid tumors are bispecific antibodies $[47,48]$.

Utomilumab (PF-05082566) is a fully human IgG2 agonist monoclonal antibody that binds to $4-1 \mathrm{BB} /$ CD137. This binding induces T-cell proliferation, production of cytokines, and inhibition of tumor growth in severely compromised immunodeficient xenograft models $[49,50]$. The drug is currently being studied in combination with anti-PD1 and anti-PD-L1 agents in the treatment of solid tumors.

Entinostat is an oral benzamide derivative that acts by selectively inhibiting class I and IV histone deacetylase. The ENCORE II study was a phase II trial evaluating the use of entinostat and exemestane in patients with advanced hormone receptor-positive breast cancer that had progressed on a prior non-steroidal aromatase inhibitor. This study demonstrated a significant improvement in progression-free and overall survival. E2112 is a double-blind phase III study evaluating the use of exemestane plus entinostat/placebo in the same population and is currently ongoing [51].

The PALLAS study is an ongoing trial evaluating the use of adjuvant palbociclib in combination with standard adjuvant endocrine therapy in patients with early hormone receptor-positive, HER2-negative breast cancer. The use of CDK4/6 inhibitors as part of adjuvant therapy will have a significant impact on future therapy options and the sequencing of their use in the metastatic and recurrent settings.

The PEARL and POLARIS studies are other ongoing studies of palbociclib.

These studies may yield additional therapeutic options in the future for patients with metastatic hormone receptor-positive breast cancer.

\section{Conclusions}

Endocrine therapy forms the cornerstone of treatment for advanced-stage hormone receptor-positive breast cancer. The development of endocrine resistance has prompted the development of a multitude of novel therapeutic options, including CDK4/6, mTOR, and PI3K inhibitors. There are many additional treatments currently in development. The optimal sequencing of therapy can be complicated and depends on different factors, including prior adjuvant therapy, disease-free interval, side effects, and patient quality of life.

\section{Abbreviations}

Al: Aromatase inhibitor; CDK4/6: Cyclin-dependent kinase 4/6; CR: Complete response; DLT: Dose-limiting toxicity; ER: Estrogen receptor; HR: Hazard ratio; ORR: Overall response rate; PFS: Progression-free-survival; SERD: Selective estrogen receptor downregulator

\section{Authors' contributions}

AD performed the literature search and compilation of the review. JL reviewed the paper and was a major contributor to the writing. DS reviewed and helped edit the paper. All authors read and approved the final manuscript.

Ethics approval and consent to participate Not applicable.

\section{Competing interests}

The authors declare that they have no competing interests.

\section{Publisher's Note}

Springer Nature remains neutral with regard to jurisdictional claims in published maps and institutional affiliations.

Received: 3 April 2018 Accepted: 22 May 2018

Published online: 11 June 2018

References

1. Reinert T, Barrios $\mathrm{CH}$. Optimal management of hormone receptor positive metastatic breast cancer in 2016. Ther Adv Med Oncol. 2015;7(6):304-20.

2. Sini V, Cinieri $S$, Conte $P$, De Laurentiis $M$, Leo AD, Tondini C, Marchetti P. Endocrine therapy in post-menopausal women with metastatic breast cancer: from literature and guidelines to clinical practice. Crit Rev Oncol Hematol. 2016;100:57-68.

3. Di Leo A, Jerusalem G, Petruzelka L, Torres R, Bondarenko IN, Khasanov R, Verhoeven D, Pedrini JL, Smirnova I, Lichinitser MR, Pendergrass K, Garnett S, Lindemann JP, Sapunar F, Martin M. Results of the CONFIRM phase III trial comparing fulvestrant $250 \mathrm{mg}$ with fulvestrant $500 \mathrm{mg}$ in postmenopausal women with estrogen receptor-positive advanced breast cancer. J Clin Oncol. 2010;28(30):4594-600.

4. Robertson JF, Lindemann JP, Llombart-Cussac A, Rolski J, Feltl D, Dewar J, Emerson L, Dean A, Ellis MJ. Fulvestrant $500 \mathrm{mg}$ versus anastrozole $1 \mathrm{mg}$ for the FIRST-line treatment of advanced breast cancer: follow-up analysis from the randomized 'FIRST' study. Breast Cancer Res Treat. 2012;136(2):503-11.

5. Ellis MJ, Llombart-Cussac A, Feltl D, Dewar JA, Jasiówka M, Hewson N, Rukazenkov Y, Robertson JF. Fulvestrant $500 \mathrm{mg}$ versus anastrozole $1 \mathrm{mg}$ for the First-line treatment of advanced breast cancer: overall survival analysis from the phase II FIRST study. J Clin Oncol. 2015;33(32):3781-7.

6. Robertson JFR, Bondarenko IM, Trishkina E, Dvorkin M, Panasci L, Manikhas A, Shparyk Y, Cardona-Huerta S, Cheung KL, Philco-Salas MJ, Ruiz-Borrego M, Shao Z, Noguchi S, Rowbottom J, Stuart M, Grinsted LM, Fazal M, Ellis MJ. Fulvestrant $500 \mathrm{mg}$ versus anastrozole $1 \mathrm{mg}$ for hormone receptor-positive advanced breast cancer (FALCON): an international, randomised, double-blind, phase 3 trial. Lancet. 2016;388(10063):2997-3005.

7. Cardoso F, Costa A, Senkus E, Aapro M, André F, Barrios CH, Bergh J, Bhattacharyya G, Biganzoli L, Cardoso MJ, Carey L, Corneliussen-James D, Curigliano G, Dieras V, El Saghir N, Eniu A, Fallowfield L, Fenech D, Francis P, Gelmon K, Gennari A, Harbeck N, Hudis C, Kaufman B, Krop I, Mayer M, Meijer H, Mertz S, Ohno S, Pagani O, et al. 3rd ESO-ESMO international consensus guidelines for advanced breast Cancer (ABC 3). Ann Oncol. 2017;28(1):16-33. 
8. Bedard PL, Freedman OC, Howell A, Clemons M. Overcoming endocrine resistance in breast cancer: are signal transduction inhibitors the answer? Breast Cancer Res Treat. 2008;108(3):307-17.

9. Clarke R, Tyson JJ, Dixon JM. Endocrine resistance in breast cancer-an overview and update. Mol Cell Endocrinol. 2015;418(Pt 3):220-34.

10. Augereau P, Patsouris A, Bourbouloux E, Gourmelon C, Abadie Lacourtoisie S, Berton Rigaud D, Soulié P, Frenel JS, Campone M. Hormonoresistance in advanced breast cancer: a new revolution in endocrine therapy. Ther Adv Med Oncol. 2017;9(5):335-46

11. Turner NC, Huang Bartlett C, Cristofanilli M. Palbociclib in hormone-receptorpositive advanced breast cancer. N Engl J Med. 2015;373(17):1672-3.

12. Barroso-Sousa R, Shapiro Gl, Tolaney SM. Clinical development of the CDK4/6 inhibitors ribociclib and abemaciclib in breast cancer. Breast Care (Basel). 2016;11(3):167-73.

13. Vidula N, Rugo HS. Cyclin-dependent kinase $4 / 6$ inhibitors for the treatment of breast cancer: a review of preclinical and clinical data. Clin Breast Cancer. 2016;16(1):8-17.

14. Xu H, Yu S, Liu Q, Yuan X, Mani S, Pestell R, Wu K. Recent advances of highly selective CDK4/6 inhibitors in breast cancer. J Hematol Oncol. 2017;10:97.

15. Finn RS, Martin M, Rugo HS, Jones S, Im SA, Gelmon K, Harbeck N, Lipatov ON, Walshe JM, Moulder S, Gauthier E, Lu DR, Randolph S, Diéras V, Slamon DJ. Palbociclib and letrozole in advanced breast cancer. N Engl J Med. 2016;375(20):1925-36

16. Rugo H, Finn R, Dieras V, Ettl J, Lipatov O, Joy A, Harbeck N, Castrellon A, Lu D, Mori A, Gauthier E, Huang C, Gelmon K, Slamon D. Palbociclib (PAL) + letrozole (LET) as first-line therapy in estrogen receptor-positive $(E R+) /$ human epidermal growth factor receptor 2-negative (HER2-) advanced breast cancer (ABC): Efficacy and safety updates with longer follow-up across patient subgroups. In: 2017 San Antonio breast Cancer symposium. San Antonio; 2017.

17. Martín M, Hoffman J, Ruiz-Borrego M, Muñoz M, Calvo L, Crownover P, García-Sáenz J, Alba E, Wang D, Thallinger C, Stradella A, Montaño Á, Adamo B, Antolín S, Moreno-Antón F, Falo C, Ruiz V, Martín N, Caballero R, Carrasco E, Gil-Gil M. Evaluation of the drug interaction potential of palbociclib and exemestane — results from the PEARL pharmacokinetic sub-Study. In: 2017 San Antonio Breast Cancer Symposium. San Antonio; 2017.

18. Tripathy D, Bardia A, Blum J, Rocque G, Wilks S, Lakhanpal S, Migas J, Cappelleri J, Perkins J, Comstock G, Wang Y. POLARIS: Palbociclib (P) in hormone receptor-positive $(\mathrm{HR}+)$ advanced breast cancer: a prospective multicenter noninterventional study. In: 2017 San Antonio Breast Cancer Symposium. San Antonio; 2017.

19. Hortobagyi GN, Stemmer SM, Burris HA, Yap YS, Sonke GS, Paluch-Shimon S, Campone M, Blackwell KL, André F, Winer EP, Janni W, Verma S, Conte P, Arteaga CL, Cameron DA, Petrakova K, Hart LL, Villanueva C, Chan A, Jakobsen E, Nusch A, Burdaeva O, Grischke EM, Alba E, Wist E, Marschner N, Favret AM, Yardley D, Bachelot T, Tseng LM, et al. Ribociclib as first-line therapy for HR-positive, advanced breast cancer. N Engl J Med. 2016;375(18):1738-48.

20. Tripathy D, Sohn J, Im S-A, Colleoni M, Franke F, Bardia A, Harbeck N, Hurvitz S, Chow L, Lee K, Campos-Gomez S, Villanueva Vazquez R, Jung K, Carlson G, Hughes G, Diaz-Padilla I, Germa C, Hirawat S, Lu Y-S. First-line ribociclib vs placebo with goserelin and tamoxifen or a non-steroidal aromatase inhibitor in premenopausal women with hormone receptor-positive, HER2-negative advanced breast cancer: Results from the randomized phase III MONALEESA-7 trial. In: 2017 San Antonio Breast Cancer Symposium. San Antonio; 2017

21. Patnaik A, Rosen Le S, Tolaney SM, Tolcher AW, Goldman JW, Gandhi L, Papadopoulos KP, Beeram M, Rasco DW, Myrand SP, Kulanthaivel P, Li L, Frenzel M, Cronier DM, Chan EM, Flaherty KT, Wen PY, Shapiro Gl. Clinical activity of LY2835219, a novel cell cycle inhibitor selective for CDK4 and CDK6, in patients with metastatic breast cancer. In: Proceedings of the 105th Annual Meeting of the American Association for Cancer Research. San Diego: American Association for Cancer Research; 2014

22. Dickler MN, Tolaney SM, Rugo HS, Cortés J, Diéras V, Patt D, Wildiers H, Hudis CA, O'Shaughnessy J, Zamora E, Yardley DA, Frenzel M, Koustenis A, Baselga J. MONARCH 1, a phase II study of abemaciclib, a CDK4 and CDK6 inhibitor, as a single agent, in patients with refractory HR+/HER2- metastatic breast cancer. Clin Cancer Res. 2017;23(17):5218-24.

23. Sledge GW, Toi M, Neven P, Sohn J, Inoue K, Pivot X, Burdaeva O, Okera M, Masuda N, Kaufman PA, Koh H, Grischke EM, Frenzel M, Lin Y, Barriga S, Smith IC, Bourayou N, Llombart-Cussac A. MONARCH 2: abemaciclib in combination with fulvestrant in women with HR+/HER2- advanced breast cancer who had progressed while receiving endocrine therapy. J Clin Oncol. 2017;35(25):2875-84.

24. Goetz MP, Toi M, Campone M, Sohn J, Paluch-Shimon S, Huober J, Park $\mathbb{H}_{\text {, }}$ Trédan O, Chen SC, Manso L, Freedman OC, Garnica Jaliffe G, Forrester T, Frenzel M, Barriga S, Smith IC, Bourayou N, Di Leo A. MONARCH 3: abemaciclib as initial therapy for advanced breast cancer. J Clin Oncol. 2017;35(32):3638-46.

25. Steelman LS, Martelli AM, Cocco L, Libra M, Nicoletti F, Abrams SL, McCubrey JA. The therapeutic potential of mTOR inhibitors in breast cancer. Br J Clin Pharmacol. 2016;82(5):1189-212.

26. Hare $\mathrm{SH}$, Harvey AJ. mTOR function and therapeutic targeting in breast cancer. Am J Cancer Res. 2017;7(3):383-404.

27. Bachelot T, Bourgier C, Cropet C, Ray-Coquard I, Ferrero JM, Freyer G, Abadie-Lacourtoisie S, Eymard JC, Debled M, Spaëth D, Legouffe E, Allouache D, El Kouri C, Pujade-Lauraine E. Randomized phase II trial of everolimus in combination with tamoxifen in patients with hormone receptor-positive, human epidermal growth factor receptor 2-negative metastatic breast cancer with prior exposure to aromatase inhibitors: a GINECO study. J Clin Oncol. 2012:30(22):2718-24.

28. Baselga J, Campone M, Piccart M, Burris HA, Rugo HS, Sahmoud T, Noguchi S, Gnant M, Pritchard Kl, Lebrun F, Beck JT, Ito Y, Yardley D, Deleu I, Perez A, Bachelot T, Vittori L, Xu Z, Mukhopadhyay P, Lebwohl D, Hortobagyi GN. Everolimus in postmenopausal hormone-receptor-positive advanced breast cancer. N Engl J Med. 2012;366(6):520-9.

29. Piccart M, Hortobagyi GN, Campone M, Pritchard Kl, Lebrun F, Ito Y, Noguchi S, Perez A, Rugo HS, Deleu I, Burris HA, Provencher L, Neven P, Gnant M, Shtivelband M, Wu C, Fan J, Feng W, Taran T, Baselga J. Everolimus plus exemestane for hormone-receptor-positive, human epidermal growth factor receptor-2-negative advanced breast cancer: overall survival results from BOLERO-2†. Ann Oncol. 2014;25(12):2357-62.

30. Jerusalem G, Mariani G, Ciruelos EM, Martin M, Tjan-Heijnen VC, Neven P, Gavila JG, Michelotti A, Montemurro F, Generali D, Simoncini E, Lang I, Mardiak J, Naume B, Camozzi M, Lorizzo K, Bianchetti S, Conte P. Safety of everolimus plus exemestane in patients with hormone-receptor-positive, HER2-negative locally advanced or metastatic breast cancer progressing on prior non-steroidal aromatase inhibitors: primary results of a phase IIIb, open-label, single-arm, expanded-access multicenter trial (BALLET). Ann Oncol. 2016;27(9):1719-25.

31. Schmid P, Zaiss M, Harper-Wynne C. MANTA - a randomized phase II study of fulvestrant in combination with the dual mTOR inhibitor AZD2014 or everolimus or fulvestrant alone in estrogen receptor-positive advanced or metastatic breast cancer. In: San Antonio Breast Cancer Symposium 2017. San Antonio; 2017.

32. Juric D, Argiles G, Burris H, Gonzalez-Angulo A, Saura C, Quadt C, Douglas M, Demanse D, De Buck S, Baselga J. Phase I study of BYL719, an alpha-specific PI3K inhibitor, in patients with PIK3CA mutant advanced solid tumors: preliminary efficacy and safety in patients with PIK3CA mutant ER-positive (ER+) metastatic breast cancer (MBC). In: Thirty-Fifth Annual CTRC-AACR San Antonio Breast Cancer Symposium. San Antonio: American Association for Cancer Research; 2012.

33. Juric D, Gonzalez-Angulo A, Burris H, Schuler M, Schellens J, Berlin J, Gupta A, Seggewiss-Bernhardt R, Adamo B, Gil-Martin M, Bootle D, Boehm M, De Buck S, Demanse D, Quadt C, Baselga J. Preliminary safety, pharmacokinetics and anti-tumor activity of BYL719, an alpha-specific PI3K inhibitor in combination with fulvestrant: Results from a phase I study. In: Thirty-Sixth Annual CTRC-AACR San Antonio Breast Cancer Symposium. San Antonio: American Association for Cancer Research; 2013.

34. Juric D, Andra F, Rugo H. Combined alpelisib (BYL719) and Fulvestrant in PIK3CA-altered or wild-type estrogen receptor-positive/human epidermal growth factor receptor 2-negative advanced breast cancer. In: 2016 Miami Breast Cancer Conference; 2016.

35. Massacesi C, Di Tomaso E, Urban P, Germa C, Quadt C, Trandafir L, Aimone P, Fretault N, Dharan B, Tavorath R, Hirawat S. PI3K inhibitors as new cancer therapeutics : implications for clinical trial design. OncoTargets Ther. 2016;9:203-10.

36. Baselga J, Im SA, Iwata H, Cortés J, De Laurentiis M, Jiang Z, Arteaga CL, Jonat W, Clemons M, Ito Y, Awada A, Chia S, Jagiełło-Gruszfeld A, Pistilli B, Tseng LM, Hurvitz S, Masuda N, Takahashi M, Vuylsteke P, Hachemi S, Dharan B, Di Tomaso E, Urban P, Massacesi C, Campone M. Buparlisib plus fulvestrant versus placebo plus fulvestrant in postmenopausal, hormone receptor-positive, HER2-negative, advanced breast cancer (BELLE-2): a 
randomised, double-blind, placebo-controlled, phase 3 trial. Lancet Oncol. 2017;18(7):904-16.

37. Di Leo A, Seok Lee K, Ciruelos E, Lønning P, Janni W, O'Regan R, Mouret Reynier M, Kalev D, Egle D, Csoszi T, Bordonaro R, Decker T, Tjan-Heijnen V, Blau S, Schirone A, Weber D, El-Hashimy M, Dharan B, Sellami D, Bachelot T. BELLE-3: A phase III study of buparlisib + fulvestrant in postmenopausal women with HR+, HER2-, aromatase inhibitor-treated, locally advanced or metastatic breast cancer, who progressed on or after mTOR inhibitor-based treatment. In: 2016 San Antonio Breast Cancer Symposium. San Antonio: Cancer Research; 2016

38. Krop IE, Mayer IA, Ganju V, Dickler M, Johnston S, Morales S, Yardley DA Melichar B, Forero-Torres A, Lee SC, de Boer R, Petrakova K, Vallentin S, Perez EA, Piccart M, Ellis M, Winer E, Gendreau S, Derynck M, Lackner M, Levy G, Qiu J, He J, Schmid P. Pictilisib for oestrogen receptor-positive, aromatase inhibitor-resistant, advanced or metastatic breast cancer (FERGI): a randomised, double-blind, placebo-controlled, phase 2 trial. Lancet Oncol. 2016;17(6):811-21.

39. Juric D, Ismail-Khan R, Campone M, García-Estévez L, Becerra C, De Boer R, Hamilton E, Mayer I, Hui R, Lathrop K, Pagani O, Asano S, Bhansali S, Zhang V, Hewes B, Munster P. Phase Ib/II study of ribociclib and alpelisib and letrozole in ER+, HER2- breast cancer: Safety, preliminary efficacy and molecular analysis. In: Thirty-Eighth Annual CTRC-AACR San Antonio Breast Cancer Symposium. San Antonio: Cancer Research; 2015.

40. Forero A, Han H, Dees E, Wesolowski R, Bardia A, Kabos P, Kern K, Perea R, Pierce K, Houk B, Rugo H. Phase Ib study to assess the safety, tolerability, and clinical activity of gedatolisib in combination with palbociclib and either letrozole or fulvestrant in women with metastatic or locally advanced/recurrent breast cancer (B2151009). In: 2017 San Antonio Breast Cancer Symposium. San Antonio; 2017.

41. Willemse PH, van der Ploeg E, Sleijfer DT, Tjabbes T, van Veelen H. A randomized comparison of megestrol acetate (MA) and medroxyprogesterone acetate (MPA) in patients with advanced breast cancer. Eur J Cancer. 1990;26(3):337-43.

42. Abrams J, Aisner J, Cirrincione C, Berry DA, Muss HB, Cooper MR, Henderson IC, Panasci L, Kirshner J, Ellerton J, Norton L. Dose-response trial of megestrol acetate in advanced breast cancer: cancer and leukemia group B phase III study 8741. J Clin Oncol. 1999;17(1):64-73.

43. Lonning PE, Taylor PD, Gun A, Iddon J. High-dose estrogen treatment in post-menopausal breast patients heavily exposed to endocrine therapy. Breast Cancer Res Treat. 2001;67(2):111-6.

44. Cardoso F, Bischoff J, Brain E, Zotano Á, Lück HJ, Tjan-Heijnen VC, Tanner M, Aapro M. A review of the treatment of endocrine responsive metastatic breast cancer in postmenopausal women. Cancer Treat Rev. 2013;39(5):457-65.

45. Liu B, Song Y, Liu D. Recent development in clinical applications of PD-1 and PD-L1 antibodies for cancer immunotherapy. J Hematol Oncol. 2017;10(1):174

46. Hu X, Huang W, Fan M. Emerging therapies for breast cancer. J Hematol Oncol. 2017;10(1):98

47. Yu S, Li A, Liu Q, Li T, Yuan X, Han X, Wu K. Chimeric antigen receptor T cells: a novel therapy for solid tumors. J Hematol Oncol. 2017:10(1):78.

48. Yu S, Li A, Liu Q, Yuan X, Xu H, Jiao D, Pestell R, Han X, Wu K. Recent advances of bispecific antibodies in solid tumors. J Hematol Oncol. 2017;10(1):155

49. Tolcher AW, Sznol M, Hu-Lieskovan S, Papadopoulos KP, Patnaik A, Rasco DW Di Gravio D, Huang B, Gambhire D, Chen Y, Thall AD, Pathan N, Schmidt EV, Chow LQM. Phase Ib study of utomilumab (PF-05082566), a 4-1BB/CD137 agonist, in combination with pembrolizumab (MK-3475) in patients with advanced solid tumors. Clin Cancer Res. 2017;23:5349-57.

50. Wang J, Yuan R, Song W, et al. PD-1, PD-L1 (B7-H1) and tumor-site immune modulation therapy: the historical perspective. J Hematol Oncol. 2017;10(1):34.

51. Sri Lakshmi HY, Zhao F, Miller KD, Tevaarwerk AJ, Wagner LI, Gray RJ, Sparano JA, Connolly RM. E2112: randomized phase iii trial of endocrine therapy plus entinostat/placebo in patients with hormone receptor-positive advanced breast cancer. NPJ Breast Cancer. 2018:4(1):1-5.

\section{Ready to submit your research? Choose BMC and benefit from:}

- fast, convenient online submission

- thorough peer review by experienced researchers in your field

- rapid publication on acceptance

- support for research data, including large and complex data types

- gold Open Access which fosters wider collaboration and increased citations

- maximum visibility for your research: over $100 \mathrm{M}$ website views per year

At BMC, research is always in progress.

Learn more biomedcentral.com/submissions 